\title{
Object Model Research Based on Data-Driven
}

\author{
Guofei Zhang ${ }^{1}$, Xiang Duª, ${ }^{2 a}$ Lu Wang $^{3}$ \\ ${ }^{1}$ Southwest Forestry University, Yunnan, Kunming, 650224, China \\ ${ }^{2}$ Kunming Metallurgy College, Yunnan, Kunming 650033, China \\ ${ }^{3}$ Academy of Educational Sciences, Yunnan, Kunming 650223
}

Key words: MIS, object model, OOA/OOD, data-driven, MDD,IFS model,four tiers model

\begin{abstract}
The paper presents a kind of OOA/OOD method about management information system (MIS). The object model includes information model,function model and status model, named IFS model in this paper. These models are controlled by some data. Model Driven Development(MDD) is a kind of model driven, which is a method of model driven software development(data-Driven).And MIS which is built through data-driven can adapt to different application areas.
\end{abstract}

\section{Introduction}

Software development has experienced four stages, which are the computing center, data center, the object center(integration of data and processing), and the developing model center . In the age of the model center, MIS development is a process of model driven by the (Model Information), which runs through the analysis, design, implementation, configuration, maintenance and management of MIS development. Model Driven Development(MDD) is a kind of model driven, which is a method of model driven software development(data-driven). The model runs through the requirement analysis, design, implementation and maintenance of the whole process of software development method [2-4].In this paper, the object model is the model of MIS, including information model, status model and function model.And MIS which is built through data-driven can adapt to different application areas.

\section{Object model}

The object model of MIS must be able to meet all kinds of the application requirements of information processing. In the development of MIS, it is very important to abstract the information characteristic, which reflects data logical structure of MIS.

${ }^{a}$ Corresponding Author:xianger_77@sina.com.cn

b.This paper is Supported by the scientific research foundation of the education department of Yunnan

Province[2015Y552] and the research foundation of Kunming College of Metallurgy[14D006] ; 
Two MIS which have the same information characteristic, have a certain degree of similarity in data description feature.And So that it is also relatively high that the realization of the program code reused of the two systems (REUSED). If we use the structural features of MIS, the use of object-oriented technology to achieve the characteristics of the program code, the development of a more flexible MIS software components, you can use the existing results from the system analysis and logic stage, to a large extent, improve the system's development efficiency.

In our research, the object model including information model, function model and status model is used to construct MIS.

\subsection{Information model}

Information model abstracted from MIS is used to describe MIS information structure.

information structure of different MIS is different, and information model is different. Figure 1 shows the structure of the personnel management information, figure 2 is the information structure of the information structure after the abstract data model obtained by Figure 1. Figure 4\&Figure 3 are another information structure and information model.

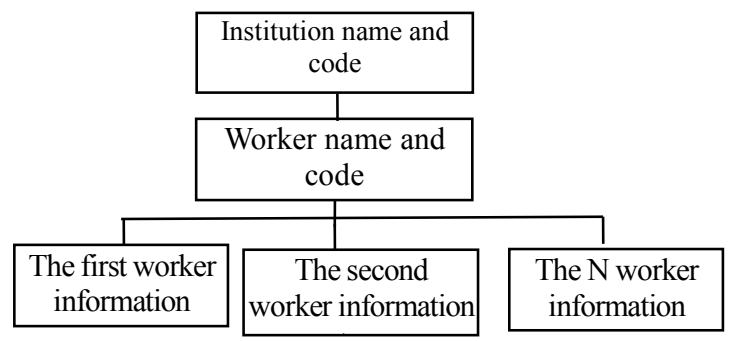

Fig1 Chart of information construction of personnel management

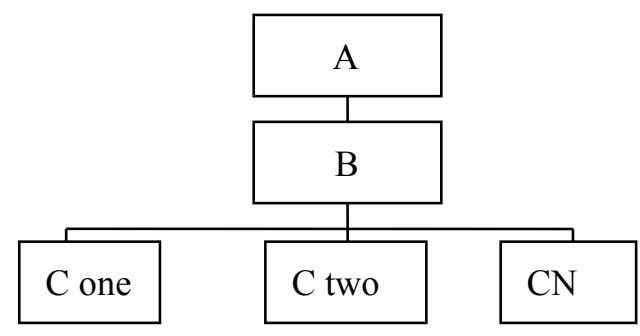

Figure 2 information Model abstracted from Figure 1

Information structure describes the structural features of MIS information data. the practical different MIS whose information structure are same can be describe by the same information model. The information structure of the student system(Figure 5) can be described by information model(Figure 2); The information structure of the course system(Figure 6) can be described by information model(Figure 4); So the same information model can describe the data characteristics of MIS with the same information structure.The logical relationship of MIS data can be described by information model.

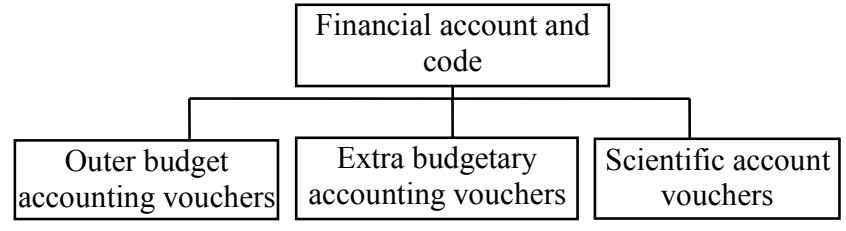

Figure 3 Chart of information construction of Financial System

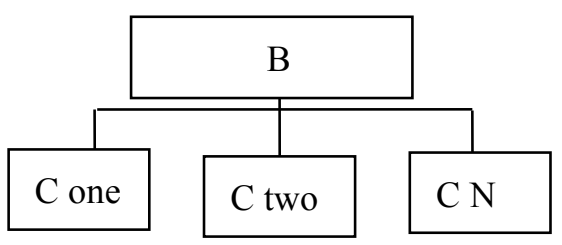

Figure 4 Information Model abstracted from Figure 3

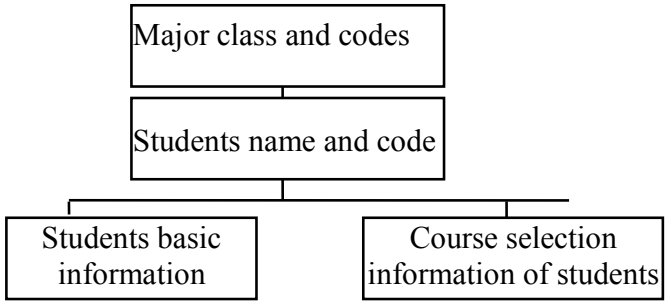

Figure 5 Chart of information construction of students System

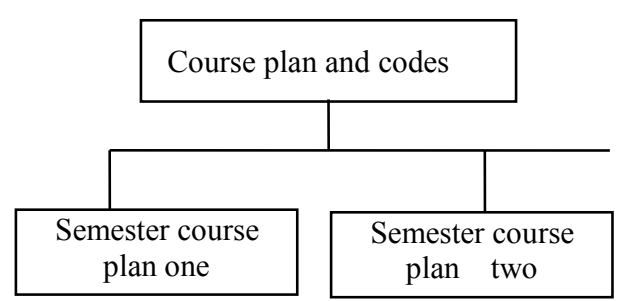

Figure 6 Chart of information construction of courses System 


\section{2 status model}

Status model is used to describe feature of MIS interface status and MIS data.

The interface status includes the representation of the human-machine interface status and the data interface.Human-machine interface status is human-machine interaction of MIS. because the information structure is different, human-machine interface is also different. Such as information model of figure 2 has three types of entities and two relations; information model of figure 4 has the two kind of entity and one relation. The 2 types of information model which are different, need to use different status model as two information model of human-computer interface carrier. In addition, even if the same information model exists in a variety of ways, a number of status models are required to meet the application requirements of different performance on the human-machine interface.

\section{3 function model}

Function model is used to describe the changes characteristics of MIS data, including the description of the data processing and the description of the system's internal data transfer mechanism.

There is a certain correlation between human-machine interface function and data processing characteristics of MIS. Although the same information model of the same status model structure, but their data processing characteristics are different. In Figure 1 and figure 3, MIS has the same information model, and can be used to implement the same interface status model for their information structure, but they are different in their data processing function at the interface between human and computer.

Another important aspect of data processing is to realize internal data conversion. Data conversion is the function of the data stream processing of MIS. MIS must provide the data output to meet the application requirements. So function model is able to provide data conversion or statistical summary, in order to meet the needs of MIS of a wide variety of data. In the actual software environment, this conversion process is started by a user or an external event. An important role for function model is to realize the description of the conversion rules, and then realize the data conversion and processing function.

\section{MIS construction principle}

We use the above three models as the basis for building MIS. Information model is the basis of the data structure and storage of MIS, and status model is the basis of MIS interface and human-computer interaction. Based on these three kinds of models, we can make full use of the existing development results in various stages of MIS development, so as to accomplish the tasks of each stage. Information model, which is based on the database technology, the logic structure of the system database is obtained by status model, and the application of the system is obtained by the combination of object-oriented technology. Function model and the object-oriented technology can obtain the application program of data conversion.

The content of information model describes the relationship between entities and entities at the stage of the design. In the design phase, it becomes the table and table of the database.

Status model is used to describe the interface of the system. He has two functions: (1) the system's data on the man-machine interface display; (2) to provide the data on the man-machine interface to implement the event. In the man-machine interface, the data must be based on information model, and the number of different data models are different, the relationship between the entities is different, so different status models are designed for different performance requirements.

Function model is used to describe the changes characteristics of MIS data. It is the behavior characteristic of MIS data. On the other hand, functional prototypes have to be linked with the status of the prototype, and the system operator can describe the interface driver function prototype, which enables the prototype to accomplish the specific data processing tasks.

\section{Principle of data-driven model}




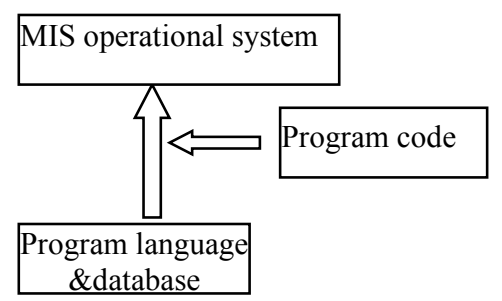

Figure 7 Chart of Two Tiers Development Model

Compared with the traditional development mode(two tiers development model,shown in figure 7),four tiers development model (figure 8) has been greatly improved. In two tiers development model, all kinds of MIS function are achieved by writing program code. When new needs arise, the MIS developed with two tiers development model only modify the source code to met.

Although the three object models are the basis of our construction of MIS, but in order to be able to use them to build a completely different business characteristics of MIS, the flexibility and adaptability of the prototype put forward higher requirements. We use the data control (control parameters) prototype method to drive the prototype, so that the prototype of the object model has a certain degree of flexibility. Since the prototype implementation is based on the object model, the final realization of the prototype is driven by the data, we call it: IFS model.

When the prototype of the object is built with data driven, we abstract the object prototype into a stable and changeable part, wherein, the logic structure of MIS is part of the stable part, and the data processing part of the system belongs to the changeable part. In addition, the description of the interface of the system is different in the different operation systems with the same information structure. When we construct object prototype, we can use the program code to realize its function, and we can use the variable (attribute) to realize its function in the program, so that the variable part of the system presents different data processing functions.

There are great differences among all kinds of systems about data processing expression. The data processing mainly includes (field, record)data increase, delete, modify, data in the voting and other basic operations. it is defined as meta operation. meta operation can achieve the basic data processing functions, and have a good reorganization.

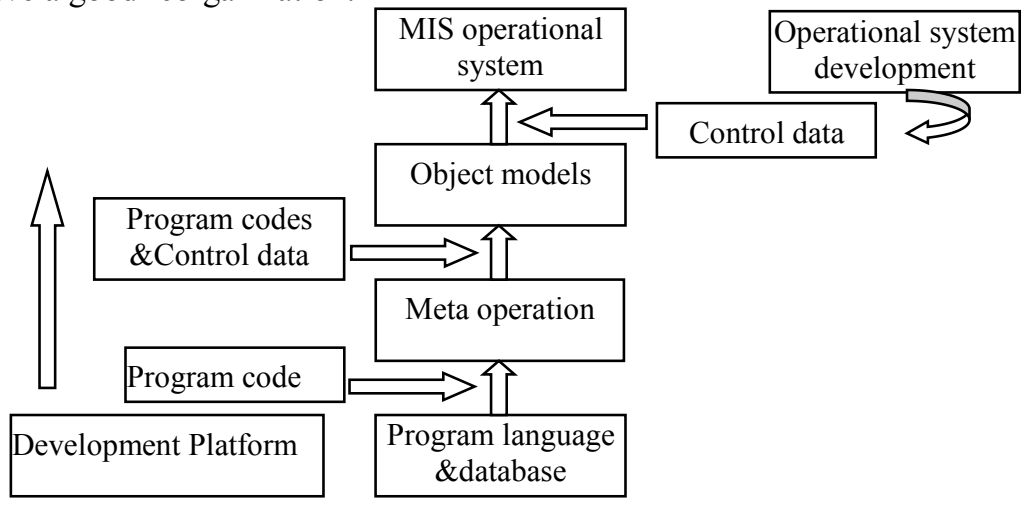

Figure8 chart of Four Tiers Development Model

For the realization of meta operation and object model programming, we construct an information processing operational system development platform, in this platform based on MIS development performance for the four tiers development model, as shown in figure 8 . The meta operation is completed by programming on the basis of programming language and database system. The stable part (structure) of object model is achieved by writing program code to achieve; the variable part of object model is achieved by control parameters(control data). in object model, meta operation is the foundation of data-driven processing function and data conversion functions. Development operational system based on the object model, he is completely driven by the data (control parameters), adjust and modify MIS, only need to modify the control parameters of the object model. 
In different MIS development, if the shortage of the existing object model to meet the needs of the real system description, in view of the abstract object model of the corresponding the program implementation, the new object model development is to program and platform provides the yuan operation as the basis, if existing meta operation can not meet the new demand, and then write new operation code.

\section{Summary}

In this paper, a kind of MMD(Model driven Development) method is found to build management information system(MIS), whose models are made by information model,status model and function model. At the software requirements analysis and preliminary design stage,information model is used to construct the logical framework of MIS data by using the exiting model. At the software program stage, status model and function model is the basis of code reuse.

Because the object model is realized by the data driven method, it has strong adaptability to the change of the application system requirement. The four tiers model of MIS development is proposed, which is based on data-driven object model.By using the four tiers model, the developer will be able to focus on the logical structure of MIS, which can make full use of the existing program foundation. In addition, software developers can develop a high level of object model and meta operation code.

\section{References}

1. Liang Jun,He Jianbang. Information Model Driven Development of MIS and Meta MIS[J], Computer science, 2003,30(3):117-119.

2. Li Liu,GuoFei Zhang.Station models research based on IFS models.Applied Mechanics and Materials:386-389

3. Li Liu,GuoFei Zhang.Research on code separation technology in B/S framework.Applied Mechanics and Materials:369-373

4. Guofei Zhang, Lu Wang, Li Liu, Xiang Du. System Model Research Based on Data-driven 2010 Asia-Pacific Youth Conference on Communication （2010 APYCC）[M],2010,102-104

5. Lu Wang, Guofei Zhang, Li Liu. Data drive OO model research of information management system[J]. Journal of Yunnan University (Natural Sciences Edition), 2009, 31 (S1): 48 51

6. Guofei Zhang. The Research of the General Forest resource MIS[D], South-West forestry university, 2007.

7. Huang Yikuan. data-driven:a practical prototyping methodology[J], Computer Development \& Applications, 1996,9 (1) :39-42

8. P. shoval, Plishin. Structured Prototyping: Integrating Prototyping into Structured system Development. Information \& Management,1998,14(3):19-30

9. Selic B. The Pragmatics of Model—driven Development[A]. IEEE Software、2003, 20(5):17-25 